\title{
Phytoprotection
}

\section{Stimulateurs des défenses naturelles des plantes : une nouvelle stratégie phytosanitaire dans un contexte d'écoproduction durable.}

\author{
II. Intérêt dles SDN en protection des cultures
}

Elicitors of natural plant defense mechanisms: a new management strategy in the context of sustainable production. II. Interest for SDN in crop protection

\section{Nicole Benhamou et Patrice Rey}

Volume 92, numéro 1, 2012

Reçu 2012-03-20; accepté 2012-05-31

URI : https://id.erudit.org/iderudit/1013299ar

DOI : https://doi.org/10.7202/1013299ar

Aller au sommaire du numéro

\section{Éditeur(s)}

Société de protection des plantes du Québec (SPPQ)

ISSN

0031-9511 (imprimé)

1710-1603 (numérique)

\section{Découvrir la revue}

\section{Citer cet article}

Benhamou, N. \& Rey, P. (2012). Stimulateurs des défenses naturelles des plantes : une nouvelle stratégie phytosanitaire dans un contexte d'écoproduction durable. II. Intérêt des SDN en protection des cultures. Phytoprotection, 92(1), 24-35. https://doi.org/10.7202/1013299ar

\section{Résumé de l'article}

$\mathrm{Au}$ cours des dernières décennies, les avancées spectaculaires de nos connaissances sur les mécanismes impliqués dans la résistance induite chez les plantes ont favorisé le développement d'initiatives visant à valoriser la stratégie de défense naturelle des plantes dans un contexte conjuguant la préservation de l'environnement et la production intensive de produits agricoles. Aujourd'hui, un nombre croissant de formulations contenant des stimulateurs des défenses naturelles (SDN) en tant que matière active arrive sur le marché et il est raisonnable de croire que de plus en plus de molécules inductrices de résistance seront disponibles dans un futur proche. Un SDN est une molécule biologique capable de déclencher les événements moléculaires, biochimiques et cytologiques menant à l'expression de la résistance chez une plante. Il s'agit donc d'une sorte de « vaccin » susceptible d'activer le « système immunitaire » de la plante de telle sorte qu'une plante initialement sensible à un agent pathogène devienne résistante. Si le chitosane est connu depuis plusieurs années, d'autres SDN d'origines variées ont récemment été découverts et certains d'entre eux ont été commercialisés. Tels sont les cas, par exemple, du Iodus $40 ®$, un polymère de $\beta$-1,3-glucanes isolé d'une algue brune, du Messenger ${ }^{\circledR}$, dont la matière active est un peptide bactérien, ou du Stifénia ${ }^{\circledR}$ qui contient des extraits de fenugrec, une légumineuse africaine. L'exploitation de la résistance induite en agriculture biologique ou en agriculture raisonnée (alternance entre la lutte biologique et la lutte chimique) est une stratégie qui offre de grandes promesses d'avenir, car elle est essentiellement fondée sur la stimulation des mécanismes naturels de défense des plantes. Il est cependant évident que des recherches sont encore nécessaires pour démontrer que cette approche 1) n'engendre aucun risque pour le consommateur (allergies ou autres désordres); 2) ne cause pas de baisses de rendement; 3 ) ne présente pas une trop grande variabilité en termes de performance; et 4) n'est pas trop onéreuse en comparaison avec une approche de lutte chimique. 


\title{
Stimulateurs des défenses naturelles des plantes : une nouvelle stratégie phytosanitaire dans un contexte d'écoproduction durable. II. Intérêt des SDN en protection des cultures
}

\author{
Nicole Benhamou' ${ }^{1}$ et Patrice Rey ${ }^{2}$
}

Reçu 2012-03-20; accepté 2012-05-31

PHYTOPROTECTION 92 : 24-35

Au cours des dernières décennies, les avancées spectaculaires de nos connaissances sur les mécanismes impliqués dans la résistance induite chez les plantes ont favorisé le développement d'initiatives visant à valoriser la stratégie de défense naturelle des plantes dans un contexte conjuguant la préservation de l'environnement et la production intensive de produits agricoles. Aujourd'hui, un nombre croissant de formulations contenant des stimulateurs des défenses naturelles (SDN) en tant que matière active arrive sur le marché et il est raisonnable de croire que de plus en plus de molécules inductrices de résistance seront disponibles dans un futur proche. Un SDN est une molécule biologique capable de déclencher les événements moléculaires, biochimiques et cytologiques menant à l'expression de la résistance chez une plante. II s'agit donc d'une sorte de "vaccin " susceptible d'activer le " système immunitaire " de la plante de telle sorte qu'une plante initialement sensible à un agent pathogène devienne résistante. Si le chitosane est connu depuis plusieurs années, d'autres SDN d'origines variées ont récemment été découverts et certains d'entre eux ont été commercialisés. Tels sont les cas, par exemple, du lodus $40^{\circledR}$, un polymère de $\beta-1,3-$ glucanes isolé d'une algue brune, du Messenger ${ }^{\circledR}$, dont la matière active est un peptide bactérien, ou du Stifénia ${ }^{\circledR}$ qui contient des extraits de fenugrec, une légumineuse africaine. L'exploitation de la résistance induite en agriculture biologique ou en agriculture raisonnée (alternance entre la lutte biologique et la lutte chimique) est une stratégie qui offre de grandes promesses d'avenir, car elle est essentiellement fondée sur la stimulation des mécanismes naturels de défense des plantes. II est cependant évident que des recherches sont encore nécessaires pour démontrer que cette approche 1) $n^{\prime}$ engendre aucun risque pour le consommateur (allergies ou autres désordres); 2) ne cause pas de baisses de rendement; 3 ) ne présente pas une trop grande variabilité en termes de performance; et 4) n'est pas trop onéreuse en comparaison avec une approche de lutte chimique.

Mots clés : Agriculture raisonnée, résistance induite, stimulateur des défenses naturelles (SDN).

[Elicitors of natural plant defense mechanisms: a new management strategy in the context of sustainable production. II. Interest for SDN in crop protection]

Over the past decades, advances made in understanding the mechanisms involved in plants' induced resistance have stimulated the development of innovative projects that focus on the natural defense strategy of plants in a context that combines environmental preservation with the massive production of agricultural products. Today, an increasing number of formulations containing elicitors of natural defenses (or SDN, for "stimulateur des défenses naturelles") as active ingredients are being marketed, and there is good reason to believe that more induced resistance-mediated molecules will be available in the near future. A SDN is a biological molecule capable of initiating the molecular, biochemical and cellular events that lead to the expression of plant resistance. As such, a SDN is similar to a vaccine in that it is capable of stimulating a plant's "immune system", thus turning a plant that was initially susceptible to a pathogen into a resistant one. If chitosan has been well known for years, other SDNs of various origins have recently been discovered, and some of them have been commercialized. Such is the case of lodus $40^{\oplus}$, a $\beta-1,3$-glucan polymer isolated form a brown alga, Messenger ${ }^{\oplus}$, whose active ingredient is a bacterial peptide, and Stifénia ${ }^{\oplus}$, which contains extracts from fenugreek, an African legume.

1. Centre de recherche en horticulture (CRH), Faculté des sciences de I'agriculture et de I'alimentation, Université Laval, Québec (Québec), Canada G1V 0A6; courriel : nicole.benhamou@fsaa.ulaval.ca

2. UMR Santé et Agroécologie du Vignoble 1065 INRA/ENITAB, Institut des Sciences de la Vigne et du Vin, Université de Bordeaux, 1, cours du Général de Gaulle, 33175 Gradignan, France 
Exploiting plants' induced resistance in biological agriculture or sustainable agriculture (alternation between biological control and chemical control) is a very promising strategy since it is essentially based on the stimulation of natural plant defense mechanisms. It is however obvious that more research is needed to demonstrate that this approach 1) does not present risks for consumers (allergies or other disorders); 2) does not affect yields; 3 ) is not too variable in terms of performance; and 4) is not too expensive compared with chemical control approaches.

Keywords: Induced resistance, SDN (stimulateurs des défenses naturelles), sustainable agriculture.

\section{INTRODUCTION}

En ce début de $X X l^{\text {e }}$ siècle, le secteur agroalimentaire fait face à un triple défi : 1) assurer la sécurité alimentaire en produisant une nourriture saine et équilibrée pour une population en croissance constante; 2) protéger l'environnement tout en maintenant une agriculture productive et écologique; et 3) composer avec la raréfaction des énergies fossiles. L'ensemble de ces considérations conduit aujourd'hui les acteurs de la profession agricole à repenser les pratiques culturales et à réévaluer l'impact des stratégies phytosanitaires sur l'innocuité des produits agricoles et sur l'environnement. Ainsi, les inquiétudes exprimées concernent non seulement les effets potentiellement néfastes des intrants chimiques sur la biodiversité intrinsèque des écosystèmes, mais également les nuisances potentielles sur la santé des consommateurs. Limitée à l'origine à une vision plutôt simpliste, c'est-à-dire la plante et son agresseur, la protection des cultures s'est progressivement étendue à l'environnement des plantes dans le but de mettre en perspective les cultures et leurs agresseurs au sein d'écosystèmes qui sont complexes de nature.

Dans ce contexte de développement d'une agriculture écologiquement intensive, l'un des premiers moyens alternatifs de lutte à avoir fait l'objet de recherches concernait I'utilisation de plantes génétiquement modifiées (Yarrow 1995). Ainsi, la culture de certaines variétés de pommes de terre (Solanum spp.) ou de courges (Curcubita spp.) commercialisées par la compagnie américaine Monsanto pour leur résistance à des infections virales est déjà autorisée aux États-Unis. Cependant, I'opinion publique européenne est globalement opposée à l'idée d'une alimentation transgénique, principalement en raison des incertitudes reliées à l'innocuité des produits issus de ces plantes pour l'humain et aux risques de pollution de l'environnement par la transmission du pollen.

La deuxième approche alternative envisagée depuis quelques décennies consiste en l'exploitation du potentiel qu'ont certains microorganismes antagonistes à interagir avec des agents pathogènes ou des insectes ravageurs (Cross et Polonenko 1996). Cette stratégie de lutte biologique est utilisée avec succès pour lutter contre certains insectes ravageurs, mais elle n'est encore que peu répandue dans un contexte de lutte contre les agents pathogènes des cultures (Harman et al. 2010). L'une des raisons expliquant les réticences du marché envers ces biopesticides concerne leur coût qui est souvent trop élevé pour être compatible avec des applications agronomiques à grande échelle. En effet, non seulement ces produits requièrent une production en masse et donc des équipements particuliers tels que des fermenteurs, mais ils nécessitent aussi une formulation bien précise pour assurer la survie des microorganismes pendant l'entreposage et pour maintenir leur activité biologique lors des traitements. Une autre contrainte majeure est liée à leur dépendance à des conditions climatiques particulières (Cross et Polonenko 1996). Malgré toutes ces contraintes, les producteurs agricoles se disent cependant prêts à utiliser des approches phytosanitaires plus respectueuses de l'environnement, en autant que celles-ci soient efficaces et disponibles à un coût raisonnable. C'est ainsi que dans un effort collectif de réduction des pesticides chimiques, plusieurs approches dites "biologiques " ont été préconisées, et ce, souvent de façon empirique. On peut citer, par exemple, I'utilisation du cuivre et du soufre, produits dits "biologiques " parce qu'ils sont naturels et donc non synthétisés chimiquement. Le cuivre a été le premier produit " biologique "proposé aux agriculteurs pour lutter contre le mildiou de la vigne, Plasmopara viticola (Berk. \& M.A. Curtis) Berl. \& De Toni in Sacc., lorsque cette maladie est apparue en Europe en 1878. Le soufre, quant à lui, a permis de lutter contre l'oïdium de la vigne, Uncinula necaton (Schwein.) Burrill, apparu en Europe en 1848. Plus d'un siècle après leurs découvertes, ces produits sont toujours utilisés, en particulier en agriculture biologique qui ne dispose pas d'autres moyens de lutte. Ces deux produits posent cependant d'importants problèmes. Le cuivre, du fait de sa dégradation très lente, peut intoxiquer les sols, tandis que l'utilisation du soufre est aujourd'hui remise en question, principalement en raison de son effet phytotoxique à des températures de $27-28{ }^{\circ} \mathrm{C}$ (températures que I'on retrouve couramment dans les serres) et de son effet négatif sur les insectes prédateurs utilisés dans le cadre de la régie des cultures en serre.

Un autre produit inorganique qui a retenu I'attention est le bicarbonate de potassium. Après un long débat sur le mode d'action de ce produit, il semble aujourd'hui acquis que l'ion bicarbonate exerce un effet antifongique direct sur les champignons pathogènes responsables de l'oïdium (Israël et al. 1993). Cependant, l'effet protecteur de ce produit ne se manifeste qu'en présence d'une faible infestation. En effet, plusieurs travaux ont démontré que le bicarbonate de potassium ne pouvait contrôler les infections sévères (plus de $50 \%$ de maladie sur les feuilles), réduisant ainsi son intérêt en tant qu'alternative aux produits de synthèse. $D^{\prime}$ autres produits biologiques, incluant des huiles (ex. : huile de Neem), des dérivés du lait (Crisp et al. 2006), des détergents, des antitranspirants et même des extraits de compost ont été proposés, mais aucun n'a pu atteindre l'efficacité des produits chimiques (Bélanger et Labbé 2002). 
Au cours des dernières décennies, les avancées spectaculaires de nos connaissances sur les mécanismes impliqués dans la résistance induite chez les plantes (Benhamou et Rey 2012) ont conduit la communauté scientifique à envisager l'exploitation de cette stratégie de défense naturelle dans un contexte conjuguant la préservation de l'environnement et la production agricole intensive (Klarzinsky et Fritig 2001). Aujourd'hui, plusieurs facteurs militent en faveur de l'utilisation des stimulateurs de défense naturelles (SDN) pour sensibiliser les plantes à répondre plus rapidement et plus efficacement à des stress biotiques et/ou abiotiques (Benhamou 2009; Lyon et al. 1995)

1. Les ingrédients actifs des SDN sont des substances entièrement biologiques ou des dérivés synthétiques de molécules biologiques;

2. Les SDN présentent l'avantage de conserver leur activité biologique sur une longue période grâce à une formulation appropriée. En général, ils ne sont pas dépendants des conditions climatiques, mais leur efficacité varie en fonction du cycle biologique de la plante.

3. Les SDN sont capables de conférer aux plantes une résistance systémique et durable.

4. Les SDN n'exercent pas de phytotoxicité répertoriée et leur toxicité pour l'environnement est réduite, voire inexistante. De plus, ils sont biodégradables.

5. Les SDN exercent un effet potentiel sur la réduction des fréquences d'apparition de phénomènes de résistance.

6. L'application des SDN est relativement simple et s'intègre parfaitement aux pratiques culturales usuelles.

7. Les SDN sont sécuritaires pour la santé humaine et animale.

Le nombre de formulations SDN homologuées croît d'une année à l'autre. II est donc raisonnable de penser que de plus en plus de molécules inductrices de résistance seront disponibles pour les agriculteurs dans un futur proche. Néanmoins, les recherches doivent être poursuivies pour mieux appréhender le mode d'action de ces molécules élicitrices. L'optimisation des formulations et une meilleure connaissance du mode et de la fréquence d'application de ces produits permettront également d'améliorer leur efficacité en serre et au champ.

\section{LES SDN : CONCEPT ET APPLICATIONS EN AGRICULTURE}

Les données de l'Observatoire de résidus de pesticides (www.observatoire-pesticides.gouv.fr) actuellement disponibles indiquent que le marché mondial des pesticides chimiques est de l'ordre de 40 milliards \$ US, alors que celui des biopesticides se situe aux alentours de 1 milliard \$ US. L'Europe est certainement le plus gros utilisateur de pesticides, principalement pour la protection du blé (Triticum L. spp.) et de la vigne (Vitis vinifera L.). De son côté, I'Amérique du Nord emploie environ $22 \%$ de la production mondiale de pesticides (incluant les herbicides et les insecticides), surtout pour le contrôle des maladies affectant les cultures maraîchères et la vigne
(Figure 1). Au Québec, I'application du Code de gestion des pesticides (en vigueur depuis avril 2003) favorise grandement le développement de nouvelles stratégies d'intervention phytosanitaires en remplacement des pesticides chimiques, alors qu'en France I'objectif du programme Écophyto 2018, faisant suite aux états généraux du Grenelle de I'environnement, prévoit une diminution de I'utilisation d'intrants chimiques de l'ordre de $50 \%$ d'ici 2018. Dans un tel contexte, l'ensemble de I'arsenal législatif et réglementaire a été réorganisé pour encadrer l'évolution incontournable des pratiques agricoles. L'agriculture et la forêt doivent non seulement produire plus pour répondre aux besoins d'une population mondiale en phase exponentielle de croissance, mais aussi produire mieux pour préserver les ressources naturelles et la biodiversité.

L'agriculture a longtemps été, et est encore, largement dépendante des méthodes de protection contre les bioagresseurs. À titre d'exemple, sans protection chimique, la culture de la vigne, des céréales ou des plantes maraîchères serait réduite d'au moins $50 \%$. Cependant, les bienfaits apparents de la lutte chimique sont devenus, au fil du temps, des sources de préoccupations majeures. L'accumulation de résidus toxiques dans la chaîne alimentaire, la contamination des eaux de surface et souterraines, le développement fréquent de résistance des agents pathogènes aux doses préconisées et les conséquences du déséquilibre engendré (ex. : éradication d'une catégorie de ravageurs suivie de l'apparition d'une autre catégorie d'ennemis des plantes cultivées encore plus agressive) sont parmi les problèmes les plus sérieux auxquels a été confrontée l'industrie agrochimique. Obligée de retirer de plus en plus de produits du marché en raison de leur toxicité démontrée, cette industrie n'a eu d'autres choix que d'en découvrir de nouveaux. II n'est plus certain aujourd'hui que la capacité d'invention de l'industrie agrochimique pourra dépasser la capacité d'adaptation des agents pathogènes (Leroux et al. 2002). Au cours des dernières années, la réglementation en matière d'homologation de pesticides tant en Europe qu'aux États-Unis s'est tellement alourdie que seules quelques grosses compagnies agrochimiques comme Syngenta ou Bayer CropScience sont encore capables d'assumer les coûts associés à la multitude de tests toxicologiques et écotoxicologiques requis

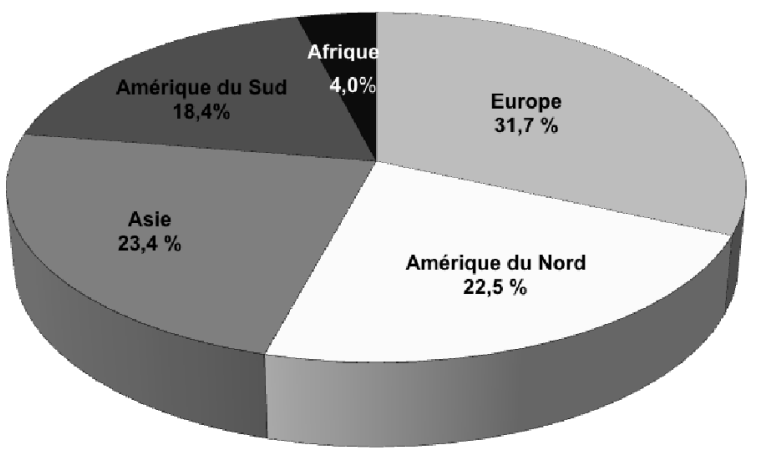

Figure 1. Pourcentage d'utilisation de pesticides en fonction de la répartition géographique. 
(Knight et al. 1997). Outre cet aspect, la diminution progressive du nombre de matières actives disponibles sur le marché aura pour conséquence une augmentation du coût de la protection phytosanitaire, en plus d'engendrer des situations où certains agents pathogènes agressifs ne seront plus contrôlés. C'est ainsi que l'idée de complémenter la lutte chimique par de nouvelles approches naturelles s'impose de plus en plus dans le contexte d'une agriculture durable.

\section{Les SDN : définition et propriétés}

Un SDN est une molécule capable d'être reconnue par la plante et de déclencher les évènements moléculaires, biochimiques et cytologiques menant à I'expression de la résistance. En d'autres termes, un SDN est une sorte de " vaccin " susceptible d'activer le " système immunitaire " du végétal, de telle sorte qu'une plante initialement sensible à un agent pathogène devienne résistante (Benhamou 2009). Parmi les molécules appartenant aux SDN, il convient cependant de distinguer les éliciteurs des potentialisateurs. Les éliciteurs sont des molécules capables de mimer l'effet d'un agent pathogène. Ils peuvent donc stimuler la stratégie défensive des plantes en I'absence de l'agresseur. En d'autres termes, les éliciteurs entraînent, une fois appliqués, des réactions de défense qu'il y ait ou non présence d'agents pathogènes. En revanche, les potentialisateurs (ex. : BABA, acide $\beta$-aminobutyrique, benzothiadiazole (BTH)) sont des molécules qui ne déclenchent que les premières étapes de la résistance induite après leur application (Kohler et al. 2002). À la suite d'une infection, ils sensibilisent la plante à répondre plus rapidement et plus efficacement à l'attaque, un phénomène appelé "Priming ". Ainsi, lors d'une infection, le BABA stimule la cascade d'évènements menant à l'établissement de la résistance, allant de la production de formes actives de l'oxygène (FAO) à I'accumulation des protéines PR (" pathogenesisrelated "), en passant par la peroxydation des lipides membranaires, la formation de callose et I'incrustation de lignine dans les parois (Cohen et al. 1994; Siegrist et al. 2000; Zimmerli et al. 2001). Certains microorganismes bénéfiques comme les champignons mycorhiziens, les "Plant Growth Promoting Fungi " (champignons stimulant la croissance végétale) et les Rhizobium spp., qui favorisent la croissance et le développement des plantes, sont également des potentialisateurs ou des phytostimulants. Les éliciteurs, tout comme les potentialisateurs, font partie de la famille des stimulateurs des défenses naturelles des plantes (SDN).

Les SDN peuvent avoir une origine végétale, animale ou microbienne. Ce sont soit des extraits bruts (extraits bruts d'algues, de sauge, de bourdaine, d'ortie, de prêle, etc.), soit des composés plus ou moins purifiés du produit d'origine, ou le résultat d'une fermentation des extraits bruts. À l'exception de quelques produits comme le lodus $40^{\circledR}$ ou le Stifénia ${ }^{\circledR}$ qui sont homologués comme stimulateurs des défenses naturelles, la plupart des autres composés sont commercialisés en tant qu'engrais ou fertilisants même s'ils prétendent, de façon plus ou moins explicite, stimuler la stratégie défensive des plantes. Pour ces produits, les problèmes concernent le plus souvent l'absence de connaissances concernant le mode d'action, les effets indésirables (phytotoxicité, écotoxicité, toxicité humaine) et l'identification de la matière active. Dans la longue liste des produits fertilisants susceptibles de stimuler les défenses naturelles des plantes et qui sont utilisés de façon empirique, nous pouvons citer :

- Algifol, un produit à base d'extraits bruts d'algues (Allemagne);

- EM5, un cocktail de microorganismes (Suisse);

- Kendal, un mélange d'extraits de plantes (8\%) et de $\mathrm{K}_{2} \mathrm{O}$ (15\%) (Allemagne);

- Rheum palmatum L., une poudre de racines de rhubarbe (Suisse);

- Frangula alnus Miller, une poudre d'écorce de bourdaine (Suisse);

- Salvia officinalis L., une tisane de sauge (1 kg ha d'ortie fraîche);

- Urtica dioica L., une tisane d'ortie $\left(1 \mathrm{~kg} \mathrm{ha}^{-1} \mathrm{de}\right.$ sauge);

- Salix viminalis L., une tisane d'osier (100 $\mathrm{g} \mathrm{ha}^{-1}$ de tiges fraîches); et

- Equisetum arvense L., une décoction de prêle (100 $\mathrm{g} \mathrm{ha}^{-1}$ ).

Les études effectuées avec ces produits non homologués conduisent toutes à la même conclusion, soit que les résultats obtenus doivent être interprétés avec précaution pour diverses raisons, incluant : 1) ces extraits de plantes sont des mélanges de molécules non identifiées et donc non caractérisées, alors rien ne prouve que certaines d'entre elles ne pourraient pas avoir un effet allergène ou toxique pour les humains, les animaux ou les insectes (Komatsu et al. 2006); 2) aucune information n'est disponible sur l'adsorption de ces molécules par la plante et sur leur influence sur la physiologie, la croissance et, ultimement, le rendement; et 3) I'influence des conditions climatiques (lessivage par la pluie, le vent, etc.) sur l'efficacité des produits n'est pas connue (Dorn et al. 2007).

\section{Homologation et commercialisation d'un SDN}

À l'instar des autres produits phytosanitaires (pesticides chimiques), tout nouveau produit de protection des plantes doit obtenir une autorisation de mise sur le marché avant de pouvoir être utilisé sur des cultures commerciales. Ce processus est long et onéreux, et la compagnie désireuse de commercialiser un nouveau produit doit fournir des informations précises et détaillées sur les caractéristiques physicochimiques et biologiques de la matière active, sur son efficacité sous diverses conditions climatiques et dans différentes zones géographiques et, enfin, sur les risques toxicologiques potentiels. Le coût d'une telle opération s'élève à plusieurs millions de dollars ou d'euros, ce qui, le plus souvent, entrave la commercialisation de nouveaux produits ayant une activité potentiellement intéressante. Par ailleurs, lorsqu'un produit biologique parvient à être homologué (comme le lodus $40^{\circ}$ ), l'autorisation de mise en marché n'est demandée, dans un premier temps, que pour des cultures à grand intérêt économique (vigne 
et céréales), laissant ainsi de côté des secteurs moins rentables économiquement comme I'horticulture et l'arboriculture. De nouvelles demandes d'homologation doivent ensuite être présentées pour que I'usage du produit s'étende à d'autres cultures et à d'autres pays. Pour certains petits fabricants qui ont la volonté de s'engager dans la voie d'une agriculture durable et écologiquement sécuritaire, le coût des opérations est le plus souvent une barrière infranchissable. Dans une telle situation, le seul moyen de contourner cette contrainte financière est de commercialiser des produits comme des "fertilisants " ou des " engrais " et d'ensuite informer les producteurs sur les autres effets potentiels des produits.

\section{Zoom sur quelques SDN actuellement disponibles sur le marché}

$\mathrm{Si}$ le chitosane est connu depuis plusieurs années (Benhamou et Thériault 1992), d'autres SDN d'origines variées ont récemment été découverts et certains d'entre eux ont été commercialisés (Tableau 1). Tel est le cas, par exemple, du lodus $40^{\circledR}$, un polymère de $\beta$-1,3-glucanes isolé d'une algue brune; du Messenger ${ }^{\circledast}$, dont la matière active est la harpine, un peptide bactérien; et du Stifénia ${ }^{\circledR}$ qui est composé d'extraits d'une légumineuse africaine, le fenugrec, dont le principe actif appartiendrait à la famille des saponines. Par ailleurs, plusieurs travaux de recherche indiquent que des composés inorganiques, comme le silicate de potassium, pourraient être considérés comme des SDN en vertu de leur capacité à promouvoir l'activité de diverses voies métaboliques, dont celle des phénylpropanoïdes (Bélanger et Labbé 2002).

\section{Le lodus $40^{\circledast}$}

Le lodus $40^{\circledR}$, dont le principe actif est la laminarine, un polymère de $\beta$-1,3-glucanes extrait de l'algue brune (Laminaria digitata), est le premier stimulant de
I'immunité végétale à avoir été commercialisé par Goëmar (France) pour lutter contre l'oïdium, Erysiphe graminis = Blumeria graminis (DC.) Speer f. sp. tritici Em. Marchal, la septoriose, Septoria tritici Desm., et le piétin-verse du blé tendre (Triticum aestivum L.). Présenté sous la forme d'un concentré soluble, le lodus $40^{\circledR}$ est appliqué au stade " épi- $1 \mathrm{~cm}$ " à raison de $1 \mathrm{~L} \mathrm{ha}^{-1}$ et protège le blé pendant $40 \mathrm{j}$, réduisant ainsi I'utilisation massive de fongicides. Devant la généralisation de la résistance des agents pathogènes aux produits chimiques, le lodus $40^{\circledR}$ est considéré comme l'alternative la plus pertinente aux triazoles (Klarzynski et al. 2000). Trois ans seulement après son homologation, plus de 100000 ha de blé étaient efficacement protégés par ce tout premier SDN pour les céréales. Récemment, Goëmar lançait deux nouveaux produits sur le marché : le lodus ${ }^{\mathrm{TM}}$

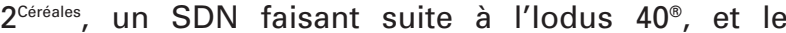
Forthia ${ }^{\circledR}$, un accélérateur de rendement des céréales. Selon un communiqué émis par Goëmar, la pénétration optimisée de la laminarine dans les tissus permet une utilisation du lodus ${ }^{\mathrm{TM}} 2^{\text {Céréales }}$ à $0,5 \mathrm{~L} \mathrm{ha}^{-1}$ contre $1 \mathrm{~L} \mathrm{ha}^{-1}$ avec le lodus $40^{\circledR}$, permettant un gain économique appréciable. Le lodus ${ }^{\mathrm{TM}} 2^{\text {Céréales }}$ est aujourd'hui homologué pour l'orge (Hordeum vulgare L.) et le blé dur (Triticum durum L.). Les recherches s'orientent maintenant vers I'utilisation de la laminarine comme principe actif de formulations destinées à protéger la fraise (Fragaria $X$ ananassa Duch. (pro sp.)) contre I'oïdium ainsi que les pommiers (Malus pumila P. Mill.) et les poiriers (Pyrus communis L.) contre le feu bactérien causé par la bactérie Erwinia amylovora (Burrill) Winslow et al.

Les mécanismes par lesquels le lodus $40^{\circledR}$ exerce un effet inducteur de résistance ont été étudiés chez le blé (Renard-Merlier et al. 2007) et la vigne (Aziz et al. 2003). Ainsi, les recherches ont montré que la protection du blé contre l'oïdium causé par Blumeria graminis f. sp. tritici était associée à une réduction

Tableau 1. Liste des SDN actuellement sur le marché

\begin{tabular}{|c|c|c|}
\hline Produit & Composition & Compagnie \\
\hline $\begin{array}{l}\text { lodus } 40^{\circledR} \text { et } \\
\text { lodus }^{\mathrm{TM}} 2^{\text {Céréales }}\end{array}$ & Laminarine $=\beta-1,3$-glucanes & Goëmar (France) \\
\hline Elexa $^{\mathrm{TM}}$ & Chitosane $=\mathrm{N}$-glucosamine & Glycogenesys Inc. (É.-U.) \\
\hline Messenger ${ }^{\oplus}$ & $\begin{array}{l}\text { Harpine = peptide produit par } \\
\text { la bactérie Erwinia amylovora }\end{array}$ & Eden Biosciences (É.-U.) \\
\hline Stifénia ${ }^{\circledast}$ & Extraits de graines de fenugrec & $\begin{array}{l}\text { Société occitane de fabrication } \\
\text { de technologies (SOFT) (France) }\end{array}$ \\
\hline Milsana $^{\oplus}$ & $\begin{array}{l}\text { Extraits de Reynoutria } \\
\text { sacchalinensis }\end{array}$ & $\begin{array}{l}\text { KHH BioSci Inc. (É.-U.) } \\
\text { Dr. Shaette (Allemagne) }\end{array}$ \\
\hline Sil-MATRIX $^{\text {TM }}$ & Silicate de potassium & PQ Corporation (É.-U.) \\
\hline $\begin{array}{l}\text { Huile de Neem } \\
{\text { (NeemAzal }{ }^{\circledR} \text {, Soluneem }}^{\circledast} \\
\text { et plusieurs autres) }\end{array}$ & $\begin{array}{l}\text { Azadirachtine extraite des graines } \\
\text { de Azadirachta indica (Neem) }\end{array}$ & $\begin{array}{l}\text { Ultrateck (Canada) } \\
\text { Lawn \& Garden Products Inc. (É.-U.) } \\
\text { et plusieurs autres sociétés dans le monde }\end{array}$ \\
\hline Cérébrosides & $\begin{array}{l}\text { Glycosphingolipides extraits de } \\
\text { la membrane de champignons }\end{array}$ & - \\
\hline Brassinostéroïdes & $\begin{array}{l}\text { Hormones végétales de type } \\
\text { stéroïde }\end{array}$ & - \\
\hline
\end{tabular}

Tableau extrait de l'ouvrage "La Résistance chez les plantes: Principes de la stratégie défensive et applications agronomiques ", N. Benhamou (2009), reproduit avec la permission des éditions Lavoisier Tec \& Doc (Paris, France). 
significative du nombre d'haustoria dans les cellules de l'épiderme et à une diminution du taux de germination des conidies. Au niveau biochimique, les auteurs rapportent une accumulation rapide de peroxyde d'hydrogène aux sites de pénétration potentielle de l'agent pathogène et une augmentation d'une activité lipoxygénase (LOX) impliquée dans la production de I'acide jasmonique. La formation de papilles aux sites de pénétration potentielle de l'agent pathogène ainsi que l'incrustation de composés phénoliques dans ces papilles font partie des réponses structurales observées à la suite d'un traitement avec le lodus $40^{\circledR}$. Aziz et al. (2003) ont rapporté qu'un traitement de la vigne avec le lodus $40^{\circledR}$ conférait une protection relativement efficace contre les champignons Botrytis cinerea (Pers.:Fr) et Plasmopara viticola. Cette protection est le résultat de la mise en place d'une cascade d'événements typiques comme la modification des flux ioniques et l'influx de $\mathrm{Ca}^{2+}$, la production de FAO, I'activation de MAP kinases, la phosphorylation de protéines, la synthèse de protéines PR (chitinases et glucanases) et la production de phytoalexines. La laminarine $n^{\prime}$ induit pas de réaction hypersensible, mais favorise plutôt l'établissement d'une résistance généralisée similaire à une résistance systémique. Son efficacité sur le blé pendant une période de $40 \mathrm{j}$ est tout à fait compatible avec les objectifs visant à limiter l'usage de produits phytosanitaires et d'engrais au strict nécessaire. Selon Goëmar, I'utilisation de la laminarine en tant que molécule stimulatrice des défenses naturelles présente de nombreux avantages : 1) il s'agit d'une molécule naturelle sans toxicité et son utilisation est sécuritaire pour l'agriculteur, le consommateur et l'environnement; 2) elle possède un large spectre $d^{\prime}$ action et elle est active sur de nombreuses espèces végétales en générant une protection efficace contre différents types d'agents pathogènes; 3) elle confère une résistance systémique, assurant ainsi la protection des organes néoformés et permet le contrôle de souches fongiques exprimant une résistance envers des pesticides chimiques; et 4) elle est compatible avec une agriculture durable et son utilisation est remarquable dans les cas de maladies dites " sans solution ", comme certaines bactérioses et viroses.

\section{Le Elexa ${ }^{T M}$}

Le Elexa ${ }^{\mathrm{TM}}$ est une formulation liquide dont le principe actif est le chitosane à une concentration de $4 \%$. Cette formulation est commercialisée par GlycoGenesys Inc. (Boston, États-Unis). II s'agit de la formulation la mieux connue même si le nombre de publications rapportant l'efficacité de ce produit est relativement restreint (Agostini et al. 2003; Jones 2000). La protection du millet perlé (Pennisetum glaucum (L.) R. Br.) contre le mildiou, Sclerospora graminicola (Sacc.) J. Schröt., a été rapportée par Sharathchandra et al. (2004). Les auteurs démontrent en effet qu'un enrobage des grains avec Elexa ${ }^{\mathrm{TM}}$ confère un niveau acceptable de protection, alors qu'une pulvérisation foliaire, ou encore mieux une combinaison des deux traitements, réduit de façon significative l'incidence de la maladie. Cependant, dans tous les cas, I'efficacité du Elexa ${ }^{\mathrm{TM}}$ est inférieure à celle du métalaxyl, un fongicide couramment utilisé sur le millet. En revanche, les auteurs notent que le Elexa ${ }^{\mathrm{TM}}$ stimule la germination des grains et la croissance des plan- tules de millet. Ces résultats indiquent que le Elexa ${ }^{\mathrm{TM}}$ est un produit dont le potentiel en tant que phytostimulant et biofongicide est bien réel. Son efficacité doit cependant être améliorée afin d'atteindre des niveaux de protection comparables à ceux obtenus avec des produits chimiques comme le métalaxyl.

\section{Le Messenger ${ }^{\oplus}$}

Le Messenger ${ }^{\circledast}$ est un produit dont la matière active est une protéine bactérienne, la harpine, qui est synthétisée à la suite de l'activité d'un gène du groupe " hrp ". Ce produit a été homologué en 2000 et il est actuellement commercialisé par EDEN BIOSCIENCE aux États-Unis. Cette compagnie américaine a réussi à insérer les gènes $h r p$ dans une souche affaiblie d'Escherichia coli afin de produire industriellement la harpine en fermenteur. En 2001, le Messenger ${ }^{\circledR}$ a reçu le prestigieux prix de la chimie verte (" Presidential Green Chemistry Award ") et a été reconnu comme étant un produit révolutionnaire (" Messenger: $A$ green chemistry revolution in plant production and food safety "). Le Messenger ${ }^{\circledR}$ est actuellement homologué pour diverses cultures, comme le coton (Gossypium spp.), la tomate (Lycopersicon esculentum Mill.), le concombre (Cucumis sativus L.), le poivron (Capsicum annuum L.), le tabac (Nicotiana tabacum L.), la fraise, le riz (Oryza sativa L.) et le froment, infectées par différents agents pathogènes.

Les mécanismes impliqués dans la protection induite par le Messenger ${ }^{\circledR}$ incluent une production accrue de FAO (particulièrement du peroxyde $d$ 'hydrogène) et une stimulation de certaines voies de signalisation, dont celles de l'acide salicylique et de l'éthylène (Reboutier et al. 2007). En dépit de ses effets bénéfiques, le fait que le Messenger ${ }^{\circledR}$ induise, dans la plupart des cas, une mort cellulaire est une contrainte majeure qu'il est indispensable de contourner en identifiant des régions bénéfiques ou délétères sur la harpine (Peng et al. 2003). Ainsi, la caractérisation récente de neuf régions fonctionnelles sur la harpine de Xanthomonas oryzae pv. oryzicola a permis de constater que chacune d'entre elles exerçait une action différente sur le riz et le tabac (Chen et al. 2008). Alors que certaines régions induisent spécifiquement une mort cellulaire, d'autres régions, comme la $\mathrm{HpaG}_{10-42}$, stimulent I'établissement d'une résistance généralisée (de type RSA) en plus de promouvoir la croissance des plantes traitées (Chen et al. 2008). L'incorporation dans la formulation du fragment $\mathrm{HpaG}_{10-42}$ au lieu du peptide complet (harpine) permettrait probablement d'améliorer l'efficacité du Messenger ${ }^{\circledast}$ en plus de lui conférer un effet de stimulateur de croissance et d'accélérateur de rendement.

\section{Le Stifénia ${ }^{\circledR}$}

La matière active du Stifénia ${ }^{\circledR}$ est un extrait de graines de fenugrec, une plante aussi appelée trigonelle ou sénégrain. Si le Stifénia ${ }^{\circledR}$ est, en premier lieu, recommandé pour la lutte contre l'oïdium de la vigne, il semble être efficace contre d'autres maladies comme la cloque du pêcher, Taphrina deformans (Berk.) Tul., et le mildiou chez diverses plantes. Homologué en 2005 par la Société Occitane de Fabrications et de Technologies (Port-la-Nouvelle, France), le Stifénia ${ }^{\circledR}$ est commercialisé depuis 2006 en France et dans 
quelques pays limitrophes comme I'Espagne, I'Italie, le Maroc et le Liban. Lors d'une étude ayant pour objectif d'évaluer l'efficacité de divers produits biologiques sur l'oïdium de la vigne, Gindro et al. (2007) ont conclu que les extraits de graines de fenugrec étaient moins efficaces que des extraits de rhubarbe ou de bourdaine. II apparaît ainsi qu'un traitement par pulvérisation du feuillage n'induit que faiblement la synthèse de phytoalexines, ne stimule pas la formation de callose aux sites de pénétration de l'agent pathogène (comme le fait le BABA, par exemple) et ne ralentit que de $40 \%$ la sporulation du champignon. Plus récemment, une étude portant sur l'évaluation de moyens de lutte contre l'oïdium du melon, Sphaerotheca fuliginae (Schltdl. Fr.) Pollacci, a apporté la preuve que le Stifénia ${ }^{\circledR}$ était nettement moins efficace que le soufre ou que des fongicides chimiques en plus d'induire des pertes de rendement significatives à la suite des applications répétées (Camele et al. 2009). Bien que l'efficacité réelle du Stifénia ${ }^{\circledR}$ fasse l'objet de controverses (Simon 2007), un avantage certain est à souligner : il semblerait qu'en traitant régulièrement une vigne avec le Stifénia ${ }^{\circledR}$, le taux d'ochratoxine, une mycotoxine produite par l'Aspergillus spp. (un champignon qui contamine les baies de raisin) et que l'on dose dans tous les vins, serait réduit de 50 \% (C. Simon, comm. pers.)

\section{Le Milsana ${ }^{\circledR}$}

Le Milsana ${ }^{\circledR}$ est une formulation biologique dont la matière active est un extrait de la renouée de sakhaline (Reynoutria sachalinensis F. Schmidt). L'extrait de $R$. sachalinensis représente $5 \%$ de la formulation. Deux composés, l'émodine et le physcion, ont été identifiés dans la matière active de la formulation. Ces composés sont des anthraquinones possédant une structure aromatique. Commercialisé à l'origine par BASF AG en Europe, ce produit est actuellement vendu par $\mathrm{KHH}$ BioSci Inc. pour son utilisation en Amérique, en Asie et en Océanie. En Europe, le Milsana $^{\circledR}$ est commercialisé par la compagnie allemande Dr. Shaette. Plusieurs articles scientifiques détaillant le mode d'action du Milsana ${ }^{\circledR}$ ont été publiés au cours des 15 dernières années (Bardin et al. 2008; Daayf et al. 1997; Fofana et al. 2005; Wurms et al. 1999). De façon générale, ce produit, lorsqu'appliqué de façon préventive, protège relativement bien les cultures maraîchères et ornementales contre l'oïdium. L'induction de gènes de défense semble être le mécanisme par lequel agit le Milsana ${ }^{\circledR}$. Daayf et al. (1997) ont été les premiers à démontrer qu'une pulvérisation de feuilles de concombre avec le Milsana ${ }^{\circledR}$ stimulait la synthèse et l'accumulation de phytoalexines, incluant des flavonoïdes à potentiel antimicrobien. Fofana et al. (2005) confirmaient, par la suite, l'importance des composés phénoliques dans la protection induite par le Milsana ${ }^{\circledR}$ en prouvant que l'inhibition de la synthèse ou de l'activité des principales enzymes de la voie de synthèse des phénylpropanoïdes (ex. : chalcone synthase) conduit à une suppression de la résistance induite par le Milsana ${ }^{\circledR}$ chez le concombre.

Les travaux de Konstantinidou-Doltsinis et al. (2006), ayant pour objectif de comparer l'efficacité du Milsana ${ }^{\circledR}$ à celle de fongicides chimiques dans la lutte contre l'oïdium de la tomate (Leveillula taurica) cul- tivée en serre, indiquent clairement que le Milsana ${ }^{\circledR}$ est substantiellement moins efficace que des produits chimiques comme les inhibiteurs de stérols qui sont des fongicides systémiques. En revanche, le Milsana ${ }^{\circledR}$ est aussi performant que le soufre, avec l'avantage de n'engendrer aucun effet délétère sur les populations d'insectes bénéfiques et de ne pas être phytotoxique à température élevée. Bardin et al. (2008) ont confirmé que le Milsana ${ }^{\circledR}$ seul était insuffisant pour combattre l'oïdium et ont démontré que son intégration avec d'autres biopesticides dans un contexte de lutte intégrée était l'approche la plus prometteuse pour réduire l'utilisation de pesticides chimiques en serre.

Parmi les autres produits susceptibles de conférer un certain niveau de résistance chez des plantes cultivées, on peut citer les suivants.

1) Le Sil-MATRIX ${ }^{\mathrm{TM}}$, un produit à base de silicate de potassium soluble qui est commercialisé par la compagnie américaine $\mathrm{PQ}$ Corporation. Ce produit est présenté comme étant un biopesticide à triple activité : fongicide, insecticide et arachnicide. Le concept d'une induction de résistance par la silice a largement été confirmé au cours des dernières années, qu'il s'agisse du melon (Cucumis melo L.) (Bi et al. 2006), de la cerise (Prunus avium L.) (Qin et Tian 2005) ou de la fraise (Kanto et al. 2007).

La silice est le deuxième élément le plus abondant sur la terre après l'oxygène (Epstein 1999). On en retrouve de grandes quantités dans la plupart des sols. Cependant, la culture intensive résulte fréquemment en une diminution de sa disponibilité pour les plantes et seule une fertilisation riche en silice peut rétablir le niveau en silice disponible et ainsi optimiser la production. La silice est un élément nutritif dit " facultatif ", car il n'est pas considéré comme essentiel à la croissance et au développement des plantes. Il est pourtant curieux de constater que la silice augmente les rendements de nombreuses cultures, qu'il s'agisse du riz, de la canne à sucre (Saccharum officinarum L.), des céréales et de certaines dicotylédones (Bélanger et al. 1995). Chez le riz, la sili-ce est absorbée en tant qu'acide monosilicilique $\left(\mathrm{Si}(\mathrm{OH})_{4}\right)$. Cette absorption est plusieurs fois supérieure à celle d'autres nutriments essentiels. Par exemple, l'accumulation de silice chez le riz est $108 \%$ plus importante que celle de l'azote (Savant et al. 1997). L'amendement en silice s'est avéré profitable pour lutter contre certaines maladies (Ou 1985). Malgré des avantages évidents pour la croissance, le développement et la protection des plantes $\mathrm{OOu}$ 1985), la silice n'a jamais été considérée à sa juste valeur dans les programmes de lutte intégrée, probablement par crainte qu'une accumulation trop importante de silice dans une plante comestible ou dans ses fruits ne nuise à sa consommation (Datnoff et al. 1997). Au cours des dernières années, de nombreux travaux de recherche ont prouvé que certaines plantes traitées avec du silicate de potassium, comme le riz et le blé, résistaient à des attaques fongiques par une activation des réactions de défense, dont notamment la production de phytoalexines de type terpénoïde (Rodrigues et al. 2004).

II est maintenant généralement admis, après des années de débats et de controverses, que la silice est un potentialisateur qui prépare la plante à réagir plus 
rapidement et plus efficacement à l'attaque par un agent pathogène. Cependant, la protection conférée est variable et c'est pourquoi une intégration de la silice avec d'autres méthodes de lutte est préférable (Datnoff et al. 2001).

2) L'huile de Neem, dont la matière active est l'azadirachtine, une substance reconnue pour ses propriétés insecticides (Helson et al. 1998). L'huile de Neem est extraite des graines produites par un arbre originaire de l'Himalaya, le Neem (Azadirachta indica L. Adelb.). Cet arbre est cultivé dans les régions tropicales ainsi que dans la région méditerranéenne. En Inde, il est considéré comme un remède universel, car tous ses organes (tiges, feuilles, racines et graines) ont des vertus thérapeutiques. Des applications foliaires d'huile de Neem sont efficaces contre plusieurs larves défoliatrices de lépidoptères ainsi que contre le charançon du pin (Pissodes strobi (Peck)) et le diprion du sapin (Neodiprion abietis (Harris)). L'huile de Neem a également des propriétés systémiques contre les mineuses et les défoliateurs forestiers lorsqu'elle est injectée dans le tronc des arbres. II est intéressant de noter que l'utilisation de l'huile de Neem à des doses efficaces contre les insectes ravageurs ne présente aucun risque pour la plupart des organismes non ciblés comme les abeilles pollinisatrices (Apis spp.), les poissons et les insectes aquatiques. Par ailleurs, ce produit se dégrade rapidement dans l'environ-nement sans laisser de résidus toxiques. En revanche, les preuves de l'efficacité de I'huile de Neem contre des agents pathogènes sont plutôt fragmentaires.

3) Les cérébrosides $\mathbf{A}$, $\mathbf{B}$ et $\mathbf{C}$, des glycosphingolipides isolés des membranes cellulaires de plusieurs champignons pathogènes (Umemura et al. 2000). Chez les plantes, les sphingolipides seraient des éliciteurs capables de stimuler la production de phytoalexines et de protéines PR et de mimer une réaction hypersensible (Umemura et al. 2000). Selon Deepak et al. (2003), I'utilisation de cérébrosides pour lutter contre le mildiou du millet perlé est une excellente alternative aux produits chimiques envers lesquels le champignon Sclerospora graminicola (Sacc.) a développé, au cours du temps, une résistance. Pour le moment, aucune formulation à base de cérébrosides n'est disponible sur le marché, probablement en raison du coût de production trop élevé.

4) Les brassinostéroïdes, des hormones de type stéroïde présentes dans tous les organes des plantes, incluant les racines (Bajguz et Tretyn 2003). De façon générale, les brassinostéroïdes stimulent la croissance des plantes, accélèrent la germination des graines, régulent la photosynthèse (Yu et al. 2004), induisent une production accrue d'éthylène et favorisent un développement précoce des fruits (Fu et al. 2008). Par ailleurs, ces composés confèrent aux plantes une résistance contre divers stress abiotiques (gel, salinité, etc.) (Nuñez et al. 2003). Des études récentes ont montré qu'un traitement avec des brassinostéroïdes protège le chou (Brassica juncea L.) contre les effets toxiques du cadmium par une production accrue d'enzymes antioxydantes (peroxydase, catalase, superoxyde dismutase) (Hayat et al. 2007).

\section{SDN et phytoprotection : avantages et inconvénients}

Ce n'est qu'avec la prise de conscience des conséquences négatives d'une agriculture dont la rationalité était trop uniquement tournée vers un objectif de production intensive qu'est apparue la nécessité de promouvoir d'autres démarches globales visant à maîtriser les quantités d'intrants (produits phytosanitaires et engrais chimiques) afin de limiter leur impact délétère sur l'environnement (Leroux et al. 2002). Dans le cadre des programmes de réduction des risques liés aux pesticides, la possibilité d'avoir accès à des SDN suscite un intérêt grandissant au sein des fédérations de producteurs horticoles et agricoles. Ces produits d'origine naturelle (Stifénia ${ }^{\circledR}$, Milsana ${ }^{\circledR}$, lodus $40^{\circledR}$, Elexa ${ }^{\mathrm{TM}}$ et Messenger ${ }^{\circledR}$ ) ont pour matière active des molécules simples qui sont isolées à partir de certaines plantes (Trigonella foenum-graecum, Reynoutria sacchalinensis) ou d'algues (Laminaria digitata), ou encore extraites de crustacés (chitosane) ou de microorganismes (harpine, cérébrosides). En vertu de leur origine biologique, ces produits ne montrent pas, a priori, un profil susceptible de présenter des dangers pour l'environnement et pour la santé des consommateurs. Au plan écotoxicologique, les effets semblent peu préoccupants, même si cet aspect doit encore être vérifié. Par ailleurs, leur biodégradabilité et les très faibles doses auxquelles ils sont appliqués font que les risques de résidus dans le sol sont relativement faibles. Ainsi, I'utilisation de ces éliciteurs naturels s'inscrit parfaitement dans le cadre d'une agriculture raisonnée pour laquelle des substances fongicides à la fois efficaces et à faible risque font cruellement défaut. II convient de mentionner, par ailleurs, que l'application abusive de fongicides conventionnels pour protéger les cultures a entraîné des contournements de résistance de la part des bioagresseurs. À force d'utiliser la même matière active contre une maladie donnée, les agents pathogènes ont eux aussi évolué, finissant par devenir résistants aux produits utilisés. En vertu de leur mode d'action indirect sur la plante et non sur l'agent pathogène et des mécanismes nombreux et variés mis en place par le végétal, la probabilité que les SDN puissent induire rapidement un contournement de résistance de la part de l'agent pathogène est extrêmement faible.

D'un point de vue pratique, de nombreux facteurs restent à définir pour que les SDN puissent atteindre une efficacité et une longévité similaires à celles des produits chimiques. Ces paramètres incluent une détermination précise des doses optimales de substances actives à utiliser et une meilleure connaissance des moments d'application, lesquels dépendent du stade physiologique de la plante, du délai de la réponse, de la durée des effets de la résistance induite et, enfin, des critères épidémiologiques du parasite. Par ailleurs, l'optimisation des formulations par l'addition d'adjuvants, tels des réducteurs de tension superficielle, ou d'agents liants est un prérequis indispensable pour leur commercialisation. Enfin, il convient de reconnaître la variabilité de l'activité biologique de certaines substances actives en fonction de facteurs environnementaux et physiologiques. Cela pose évidemment le problème de la place à réserver à ces produits biologiques dans les 
stratégies conventionnelles de lutte. Ils peuvent, dans un premier temps, s'inscrire en tant qu'agents complémentaires de la lutte chimique et peuvent jouer un rôle stratégique dans le cadre d'une approche d'antirésistance aux fongicides (Creemers 2001). II est aussi possible de les utiliser en synergie avec des fongicides conventionnels (stratégie employée avec le lodus $40^{\circledR}$ ), ce qui permettrait de réduire au moins de moitié les doses de fongicides utilisées. Par ailleurs, on peut aussi envisager d'utiliser ce type de produits à des périodes où les risques d'infections sont plus faibles.

Un autre questionnement fondamental concerne les répercussions potentielles d'un traitement des cultures avec des SDN sur la santé humaine. En effet, la stimulation du "système immunitaire " des plantes provoque l'activation d'une série de réactions biochimiques qui aboutissent à la synthèse de nouvelles molécules organiques, dont notamment des protéines PR et des phytoalexines. Pour Lyon et Newton (1997), les protéines PR sont des molécules communes à toutes les plantes et leur production accrue sous l'action d'un éliciteur ne devrait pas poser de problèmes majeurs. Cependant, plusieurs études, dont celles d'Ebner et al. (2001) et, plus récemment, d'Andersen et al. (2011), soulignent le pouvoir allergène de certaines protéines $P R$, notamment celui des PR10 qui sont des protéines proches des ribonucléases; cet aspect mérite d'être plus précisément examiné. Concernant l'accumulation de phytoalexines chez les plantes élicitées, certains auteurs s'accordent à dire qu'elle serait plutôt bénéfique pour le consommateur. En effet, plusieurs composés phénoliques, notamment les flavonoïdes, présentent des vertus thérapeutiques reconnues telles des activités anticancéreuses, antimicrobiennes, anti-inflammatoires et/ou cardio-protectrices (Ebehardt et al. 2000; King et Young 1999). On peut donc penser que les fruits et légumes issus de cultures traitées avec des SDN seraient un peu plus riches en composés phénoliques et présenteraient une plus-value au plan diététique (Treutter 2000); de tels bienfaits restent toutefois à être démontrés. Enfin, les lectines de plante sont généralement bénéfiques pour la santé humaine (Keyaerts et al. 2007; Lam et $\mathrm{Ng}$ 2011). Cependant, elles peuvent s'avérer toxiques lorsque leur concentration est trop élevée.

Un autre aspect qui mérite d'être soulevé concerne la diversité des appellations retrouvées sur le marché. Il est en effet difficile de s'y retrouver entre " vaccin des plantes ", " biostimulant ", " phytostimulant ", " stimulateur des défenses " ou même " biofongicide " pour décrire un SDN. Face au flou artistique autour de l'utilisation de ces produits souvent présentés comme fertilisants foliaires, le réseau INDRES (pour INduction De RESistance), composé d'une vingtaine de scientifiques français qui étudient les mécanismes de défense chez les plantes et le mode d'action des éliciteurs, ainsi qu'un groupe de travail se penchant sur les " intrants alternatifs ou complémentaires à la protection chimique " ont proposé que le terme SDP (stimulateur de défense des plantes) remplace le terme SDN afin d'y inclure les produits de synthèse comme le BTH ou le Fosétyl-Al (Aliette) qui ont la capacité de stimuler le " système immunitaire " des plantes. Ces chercheurs travaillent également à limiter la commercialisation de produits empiriques pour lesquels aucune donnée scientifique n'est disponible.

\section{Perspectives d'avenir des SDN en agriculture}

Aujourd'hui, les techniques culturales incorporant des approches de lutte intégrée (combinaison de lutte chimique raisonnée et de lutte biologique) s'implantent de plus en plus dans de nombreux pays (Goldenman et Wattiez 2001). Parallèlement, la prise de conscience des consommateurs envers l'impact des produits chimiques sur leur santé fait en sorte que la demande pour des produits issus de l'agriculture biologique est en progression fulgurante. Cette nouvelle dynamique du secteur agroalimentaire n'a pas échappé à l'industrie phytochimique qui se préoccupe beaucoup plus qu'auparavant de mettre au point des produits de lutte plus sécuritaires, comme des molécules d'origine naturelle (huile de Neem), des biopesticides (formulés à base de microorganismes) et des produits éliciteurs de résistance (SDN) (Copping et Menn 2000; Klarzynski et Fritig 2001). Ces derniers trouvent parfaitement leur place dans la mouvance actuelle, car ils sont dotés d'une bonne image de marque par les firmes commerciales qui les désignent sous le vocable de " vaccins des plantes " ou même de "révolution de la chimie verte ". Cependant, les nombreux avantages associés à l'utilisation de tels produits (ex. : effet systémique dans les plantes, large spectre d'action, lutte contre des maladies bactériennes et virales pour lesquelles peu de moyens de protection sont disponibles, compatibilité écologique, compatibilité avec les pratiques culturales usuelles, etc.) ne doivent pas occulter certains inconvénients que les recherches des prochaines années devront contourner. Parmi les principaux problèmes liés à l'utilisation des SDN, la variabilité en termes de performance est certainement le facteur le plus préoccupant. Viennent ensuite le temps requis pour que la résistance s'exprime, le coût énergétique lié à l'induction de résistance qui, éventuellement, pourrait se traduire par une légère baisse de rendement et, enfin, le potentiel allergène de certaines molécules de défense, comme les protéines PR10 (Ebner et al. 2001).

Dans un contexte où les préoccupations environnementales sont de plus en plus présentes, l'agriculture raisonnée devrait permettre aux producteurs agricoles de s'engager dans une démarche de développement durable en assurant une production de qualité tout en intégrant des valeurs fondamentales parmi lesquelles se retrouvent le respect de I'environnement, la maîtrise des risques sanitaires et la sécurité au travail. Sur le plan de la protection des cultures, l'agriculture raisonnée permet de restreindre au maximum les apports de pesticides grâce à une meilleure connaissance des seuils d'intervention, à I'utilisation de pièges ou à la consultation de modèles de prévision. En plus des mesures préventives, prophylactiques et culturales, ce type d'agriculture doit également faire appel à de nouvelles stratégies d'intervention phytosanitaire avec l'introduction, par exemple, de nombreux auxiliaires pour lutter contre les insectes ravageurs. En ce qui concerne les maladies, la lutte biologique au moyen d'organismes 
antagonistes et la stimulation du " système immunitaire " des plantes par les SDN sont des approches phytosanitaires qui offrent de grandes promesses $d^{\prime}$ avenir parce qu'elles répondent aux attentes grandissantes en termes de protection de l'environnement et de la santé humaine.

\section{CONCLUSION}

Depuis de nombreuses années, l'agriculteur cherche à préserver ses cultures de toutes les agressions biotiques afin de maintenir le rendement à un maximum permis par les contraintes climatiques. Même s'il n'existe pas encore de solution idéale, le plus grand défi se situe au niveau du développement d'alternatives aptes à réduire la dépendance du secteur agricole envers les pesticides. Cette lutte contre les bioagresseurs doit donc se raisonner en fonction de critères économiques, toxicologiques et environnementaux. En effet, dans un contexte où les préoccupations environnementales sont de plus en plus fortes, l'agriculture raisonnée permet d'engager l'exploitation agricole dans une démarche de développement durable en assurant une production de qualité tout en intégrant non seulement le respect de l'environnement, mais également la maîtrise des risques sanitaires ainsi que la sécurité au travail des salariés et le bien-être animal. Ainsi, en agriculture raisonnée, pour diminuer les risques pour I'humain et pour l'environnement liés à l'utilisation des produits phytosanitaires, l'agriculteur devrait déclencher un traitement uniquement lorsque la situation l'impose et en utilisant des produits autorisés aux doses homologuées. En plus des mesures préventives, prophylactiques et culturales, ce type d'agriculture doit également faire appel à de nouvelles stratégies d'intervention phytosanitaire avec l'introduction, par exemple, de nombreux auxiliaires comme les coccinelles (Coccinella spp.) pour lutter contre les insectes ravageurs. Pour les maladies, la lutte biologique au moyen d'organismes antagonistes (ex. : Trichoderma spp.) et, plus récemment, la stimulation du " système immunitaire " des plantes sont des approches qui retiennent de plus en plus l'attention. Dans ce contexte, la découverte de molécules inductrices de résistance chez les plantes, les SDN, permet d'envisager avec optimisme l'implantation de nouvelles stratégies d'intervention phytosanitaire. De telles approches, qui doivent combiner efficacité, fiabilité et sécurité environnementale, sont de plus en plus populaires et bon nombre de compagnies, incluant des compagnies connues pour commercialiser des pesticides chimiques (Syngenta, Bayer CropScience), développent des programmes de R\&D dans ce domaine.

Les avantages évidents des SDN n'en font pas pour autant une panacée et des recherches approfondies sont encore nécessaires pour élucider certaines zones d'ombre concernant principalement le temps requis pour que s'exprime pleinement la résistance, la durabilité des effets de la résistance induite, la dose optimale de matière active nécessaire et l'impact potentiel sur la santé humaine. S'il est vrai que les SDN n'exercent aucune activité toxique directe, il est possible que la stimulation des mécanismes de défense de la plante conduise à la production massive de molécules (ex. : protéines de stress) susceptibles de causer des allergies ou d'autres problèmes de santé.

Les données récentes sur la grande famille des pesticides représentant le " tout chimique " en agriculture sont édifiantes : la France est le premier consommateur européen de pesticides et le troisième à l'échelle mondiale avec environ 80000 tonnes de substances actives par an. Au niveau des fruits et légumes, on a retrouvé plusieurs résidus de pesticides interdits (endosulfan, parathion-éthyl, dialifos). Alors que I'on continue de préconiser qu'il faut manger des fruits et des légumes, plus de $48 \%$ de ces produits issus de l'agriculture sont contaminés par des pesticides, avec $4 \%$ d'entre eux se situant audessus des limites maximales en résidus (LMR). Face à une telle situation, les SDN représentent indiscutablement une solution alternative intéressante. Comme tout produit phytosanitaire, les SDN doivent cependant passer par une autorisation de mise sur le marché (AMM) pour être commercialisés, une procédure qui est lourde et onéreuse. $C^{\prime}$ est peut-être la raison pour laquelle aucune homologation de SDN n'a vu le jour au cours des 5 dernières années alors que dans la littérature scientifique nous savons qu'il existe une variété de molécules naturelles inductrices de résistance chez les plantes. Malgré tout, les recherches se poursuivent activement à travers le monde et il reste à espérer que l'objectif " zéro pesticide " soit un jour atteint.

\section{RÉFÉRENCES}

Agostini, J.P., P.M. Bushong et L.W. Timmer. 2003. Greenhouse evaluation of products that induce host resistance for control of scab, melanose, and Alternaria brown spot of citrus. Plant Dis. 87 : 69-74.

Andersen, M.B.S., S. Hall et L.O. Dragsted. 2011. Identification of European allergy patterns to the allergen families PR-10, LTP, and profilin from Rosaceae fruits. Clin. Rev. Allergy Immunol. 41 : 4-19.

Aziz, A., B. Poinssot, X. Daire, M. Adrian, A. Bézier, B. Lambert, J.M. Joubert et A. Pugin. 2003. Laminarin elicits defense responses in grapevine and induces protection against Botrytis cinerea and Plasmopara viticola. Mol. Plant-Microbe Interact. 16 : 1118-1128.

Bajguz, A. et A. Tretyn. 2003. The chemical structures and occurrence of brassinosteroids in plants. Pages 1-44 dans S. Hayat et A. Ahmad (eds.), Brassinosteroids: Bioactivity and Crop Productivity. Kluwer Academic Publishers, Dordrecht, Pays-Bas.

Bardin, M., J. Fargues et P.C. Nicot. 2008. Compatibility between biopesticides used to control grey mould, powdery mildew and whitefly on tomato. Biocontrol 46 : 476-483.

Bélanger, R.R. et C. Labbé. 2002. Control of powdery mildews without chemicals: prophylactic and biological alternatives for horticultural crops. Pages 256-267 dans R.R. Bélanger, W.R. Bushnell, A.J. Dik et T.L.W. Carver (eds.). Powdery Mildew: A Comprehensive Treatise. APS Press, St. Paul, MN, É.-U.

Bélanger, R.R., P.A. Bowen, D.L. Ehret et J.G. Menzies. 1995. Soluble silicon: its role in crop and disease management of greenhouse crops. Plant Dis. 79 : 329-336.

Benhamou, N. 2009. La Résistance chez les plantes: Principes de la stratégie défensive et applications agronomiques. Lavoisier, France. 376 p. 
Benhamou, N. et P. Rey. 2012. Stimulateurs des défenses naturelles des plantes : une nouvelle stratégie phytosanitaire dans un contexte d'éco-production durable. . Principes de la résistance induite. Phytoprotection 92 : $1-23$.

Benhamou, N. et G. Thériault. 1992. Treatment with chitosan enhances resistance of tomato plants to the crown and root rot pathogen Fusarium oxysporum f. sp. radicislycopersici. Physiol. Mol. Plant Pathol. 41 : 3352.

Bi, Y., S.P. Tian, Y.R. Guo, Y.H. Ge et G.Z. Qin. 2006. Sodium silicate reduces postharvest decay of Hami melons: Induced resistance and fungistatic effects. Plant Dis. 90 : 279-283.

Camele, I., G. Campanelli, V. Ferrari, G. Viggiani et V. Candido. 2009. Powdery mildew control and yield response of inodorus melon. Italian J. Agron. 4 : 19-26.

Chen, L., J. Qian, S. Qu, J. Long, Q. Yin, C. Zhang, X. Wu, F. Sun, T. Wu, M. Hayes, S.V. Beer et H. Dong. 2008. Identification of specific fragments of $\mathrm{HpaG}_{x o o c}$, a harpin from Xanthomonas oryzae pv. oryzicola, that induce disease resistance and enhance growth in plants. Phytopathology $98:$ 781-791.

Cohen, Y., T. Niderman, E. Mösinger et R. Fluhr. 1994. $\beta$-aminobutyric acid induces the accumulation of pathogenesis-related proteins in tomato (Lycopersicon esculentum L.) plants and resistance to late blight infection caused by Phytophthora infestans. Plant Physiol. $104: 59-66$.

Copping, L.G. et J.J. Menn. 2000. Biopesticides: a review of their action, applications and efficacy. Pest Manag. Sci. $56: 651-676$.

Creemers, P. 2001. Alternative control methods of fungal diseases: induced resistance with natural products and biological control of post-harvest diseases with yeasts. Pages 23-24 dans Protection biologique et alternative pomme : verger conservation. Colloque européen pomme, Centre Technique Interprofessionnel des Fruits et Légumes, Paris, France.

Crisp, P., T.J. Wicks, G. Troup et E.S. Scott. 2006. Mode of action of milk and whey in the control of grapevine powdery mildew. Aust. J. Plant Pathol. 35 : 487-493.

Cross, J.V. et D.R. Polonenko. 1996. An industry perspective and commercialization of biocontrol agents in Canada. Can. J. Plant Pathol. 18 : 446-454.

Daayf, F., A. Schmitt et R.R. Bélanger. 1997. Evidence of phytoalexins in cucumber leaves infected with powdery mildew following treatment with leaf extracts of Reynoutria sachalinensis. Plant Physiol. 113 : 719-727.

Datnoff, L.E., C.W. Deren et G.H. Snyder. 1997. Silicon fertilization for disease management of rice in Florida. Crop Prot. 16 : 525-531.

Datnoff, L.E., K.W. Seebold et F.J. Correa. 2001. The use of silicon for integrated disease management: reducing fungicide applications and enhancing host plant resistance. Pages 171-184 dans L.E. Datnoff, G.H. Snyder et G.H. Korndörfer (eds.). Silicon in Agriculture. Studies in Plant Science 8, Elsevier, New York, É.-U

Deepak, S.A., S. Niranjan Raj, K. Umemura, T. Kono et H. Shekar Shetty. 2003. Cerebroside as an elicitor for induced resistance against the downy mildew pathogen in pearl millet. Ann. Appl. Biol. 143 : 169-173.

Dorn, B., T. Musa, H. Krebs, P. Fried et H. Forrer. 2007. Control of late blight in organic potato production: evaluation of copper-free preparations under field, growth chamber, and laboratory conditions. Eur. J. Plant Pathol. $119: 217-240$

Ebehardt, M.V., C.Y. Lee et R.H. Liu. 2000. Antioxidant activity of fresh apples. Nature $405: 903-904$

Ebner, C., K. Hoffmann-Sommergruber et H. Breiteneder. 2001. Plant food allergens homologous to pathogenesisrelated proteins. Allergy $56: 43-44$

Epstein, E. 1999. Silicon. Annu. Rev. Physiol. Plant Mol. Biol. $50: 641-664$.
Fofana, B., N. Benhamou, D.J. McNally, C. Labbé, A. Séguin et R.R. Bélanger. 2005. Suppression of induced resistance in cucumber through disruption of the flavonoid pathway. Phytopathology 95 : 114-123.

Fu, F.Q., W.H. Mao, K. Shi, Y.H. Zhou, T. Asami et J.Q. Yu. 2008. A role of brassinosteroids in early fruit development in cucumber. J. Exp. Bot. $59: 2299-2308$.

Gindro, K., S. Godard, I. De Groote et O. Viret. 2007. Peut-on stimuler les mécanismes de défense de la vigne? Une nouvelle méthode pour évaluer le potentiel des éliciteurs. Rev. Suisse Vit. Arboric. Hortic. 39 : 377-383.

Goldenman, G. et C. Wattiez. 2001. A campaign for pesticide use reduction in Europe. Pestic. News 51 : 10-11.

Harman, G.E., M.A. Obregón, G.J. Samuels et M. Lorito. 2010. Changing models for commercialization and implementation of biocontrol in the developing and the developed world. Plant Dis. 94 : 928-939.

Hayat, S., B. Ali, S. Aiman-Hasan et A. Ahmad. 2007. Brassinosteroid enhanced the level of antioxidants under cadmium stress in Brassica juncea. Environ. Exp. Bot. 60 : 33-41.

Helson, B.V., P. de Groot, J.W. McFarlane, B.F. Zylstra et T.A. Scarr. 1998. Leader and systemic applications of neem EC formulations for control of white pine weevil (Coleoptera: Curculionidae) on jack pine and white pine. Proc. Entomol. Soc. Ont. 129 : 107-113.

Israël, H.W., S.J. Ingalls, L.L. Porter et R.K. Horst. 1993. Control of powdery mildew of rose with bicarbonates: III. A microscopic study of protection. (Abstr.). Phytopathology $83: 244$.

Jones, R. 2000. Formulating new options. Fruit Growers, Meister Publications, Boston, MA, É.-U.

Kanto, T., K. Maekawa et M. Aino. 2007. Suppression of conidial germination and appressorial formation by silicate treatment in powdery mildew of strawberry. J. Gen. Plant Pathol. 73 : 1-7.

Keyaerts, E., L. Vijgen, C. Pannecouque, E. Van Damme, W. Peumans, H. Egberink, J. Balzarini et M. Van Ranst. 2007. Plant lectins are potent inhibitors of coronaviruses by interfering with two targets in the viral replication cycle. Antivir. Res. 75 : 179-187.

King, A. et G. Young. 1999. Characteristics and occurrence of phenolic phytochemicals. J. Am. Diet. Assoc. 99 : 213218.

Klarzynski, O. et B. Fritig. 2001. Stimulation des défenses naturelles des plantes. C. R. Acad. Sci. Paris, Ser. III 324 : 953-963.

Klarzynski, O., B. Plesse, J.M. Joubert, J.C. Yvin, M. Kopp, B. Kloareg et B. Fritig. 2000. Linear $\beta-1,3$ glucans are elicitors of defense responses in tobacco. Plant Physiol. 124 : 1027-1037.

Knight, S.C., V.M. Anthony, A.M. Brady, A.J. Greenland, S.P. Heaney, D.C. Murray, K.A. Powell, M.A. Schultz, C.A. Spinks, P.A. Worthington et D. Youle. 1997. Rational and perspectives on the development of fungicides. Annu. Rev. Phytopathol. 35 : 349-372.

Kohler, A., S. Schwindling et U. Conrath. 2002. Benzothiadiazole-induced priming for potentiated responses to pathogen infection, wounding, and infiltration of water into leaves requires the NPR1/NIM1 gene in Arabidopsis. Plant Physiol. 128 : 1046-1056.

Komatsu, K., Y. Nagayama, K. Tanaka, Y. Ling, S.Q. Cai, T. Omote et M.R. Meselhy. 2006. Comparative study of chemical constituents of rhubarb from different origins. Chem. Pharmaceut. Bull. $54:$ 1491-1499.

Konstantinidou-Doltsinis, S., E. Markellou, A.M. Kasselaki, M.N. Fanouraki, C.M. Koumaki, A. Schmitt, A. LiopaTsakalidis et N.E. Malathrakis. 2006. Efficacy of Milsana ${ }^{\circledR}$ a formulated plant extract from Reynoutria sachalinensis, against powdery mildew of tomato (Leveillula taurica). Biocontrol $51: 375-392$ 
Lam, S.K. et T.B. Ng. 2011. Lectins: production and practical applications. Appl. Microbiol. Biotechnol. 89 : 45-55.

Leroux, P., R. Delorme et P. Gaillardon. 2002. Évolution des produits phytosanitaires à usages agricoles. II - Les fongicides. Phytoma $545: 8-15$.

Lyon, G.D. et A.C. Newton. 1997. Do resistance elicitors offer new opportunities in integrated disease control strategies? Plant Pathol. 46 : 636-641.

Lyon, G.D., T. Reglinski et A.C. Newton. 1995. Novel disease control compounds: the potential to "immunize" plants against infection. Plant Pathol. 44 : 407-427.

Nunez, M., P. Mazzafera, L.M. Mazorra, W.J. Siqueira et M.A.T. Zullo. 2003. Influence of brassinosteroid analogue on antioxidant enzymes in rice grown in culture medium with $\mathrm{NaCl}$. Biol. Plant. $47: 67-70$.

Ou, S.H. 1985. Rice Diseases, 2nd Edition. Commonwealth Mycological Institute, Kew, Surrey, G.-B.

Peng, J.L., H.S. Dong, H.P. Dong, T.P. Delaney, B.M. Bonasera et S.V. Beer. 2003. Harpin-elicited hypersensitive cell death and pathogen resistance require the NDR1 and EDS1 genes. Physiol. Mol. Plant Pathol. 62 : 317-326.

Qin, G.Z. et S.P. Tian. 2005. Enhancement of biocontrol activity of Cryptococcus laurentii by silicon and the possible mechanisms involved. Phytopathology $95: 69-75$

Reboutier, D., C. Frankart, J. Briand, B. Biligui, S. Laroche, J.P. Rona, M.A. Barny et F. Bouteau. 2007. The HrpNEa harpin from Erwinia amylovora triggers differential responses on the nonhost Arabidopsis thaliana cells and on the host apple cells. Mol. Plant-Microbe Interact. 20 : 94-100.

Renard-Merlier, D., B. Randoux, E. Nowak, F. Farcy, R. Durand et P. Reignault. 2007. Iodus 40, salicylic acid, heptanoyl salicylic acid and trehalose exhibit different efficacies and defence targets during a wheat/powdery mildew interaction. Phytochemistry $68: 1156-1164$.

Rodrigues, F.A., D.J. McNally, L.E. Datnoff, J.B. Jones, C. Labbé, N. Benhamou, J.G. Menzies et R.R. Bélanger. 2004. Silicon enhances the accumulation of diterpenoid phytoalexins in rice: A potential mechanism for blast resistance. Phytopathology $94: 177-183$.
Savant, N.K., G.H. Snyder et L.E. Datnoff. 1997. Silicon management and sustainable rice production. Pages 151-199 dans D.L. Sparks (ed.). Advances in Agronomy. Academic Press, New York, É.-U.

Sharathchandra, R.G., S. Niranjan Raj, N.P. Shetty, K.N. Amruthesh et H.S. Shetty. 2004. A chitosan formulation Elexa $^{\mathrm{TM}}$ induces downy mildew disease resistance and growth promotion in pearl millet. Crop Prot. 23 : 881888.

Siegrist, J., M. Orober et H. Buchenauer. 2000. $\beta$-aminobutyric acid-mediated enhancement of resistance in tobacco to tobacco mosaic virus depends on the accumulation of salicylic acid. Physiol. Mol. Plant Pathol. 56 : 95-106.

Simon, C. 2007. Stifénia doit faire ses preuves. Revue La Vigne $191: 79$.

Treutter, D. 2000. Induced resistance in plant pathology Consequences for the quality of plant foodstuffs? J. Appl. Bot. $74: 1-4$

Umemura, K., N. Ogawa, T. Yamauchi, M. Iwata, M. Shimura et J. Koga. 2000. Cerebroside elicitors found in diverse phytopathogens activate defense responses in rice plants. Plant Cell Physiol. 41 : 676-683.

Wurms, K., C. Labbé, N. Benhamou et R.R. Bélanger. 1999. Effect of Milsana and benzothiadiazole on the ultrastructure of powdery mildew haustoria in cucumber. Phytopathology 89 : 728-736.

Yarrow, S.A. 1995. Biotechnology and the plant protection program. Can. J. Plant Pathol. 17 : 277-278.

Yu, J.Q., L.F. Huang, W.H. Hu, Y.H. Zhou, W.H. Mao, S.F. Ye et S. Nogues. 2004. A role for brassinosteroids in the regulation of photosynthesis in Cucumis sativus. J. Exp. Bot. $55: 1135-1143$.

Zimmerli, L., J.P. Métraux et B. Mauch-Mani. 2001. $\beta$-aminobutyric acid-induced protection of Arabidopsis against the necrotrophic fungus Botrytis cinerea. Plant Physiol. 126 : 517-523. 